\title{
Aktuelle Trends in Diagnosestellung und Therapie der Spondylitis ankylosans - Ergebnisse einer Patienten- befragung
}

\author{
Current Trends in the Therapy for Ankylosing Spondylitis - Results of a Patient \\ Survey
}

Autoren

Institute
E. Feldtkeller ${ }^{1}$, L. Hammel ${ }^{2}$, H. Kellner ${ }^{3}$

Ankylosing Spondylitis International Federation, Vizepräsident

Deutsche Vereinigung Morbus Bechterew e. V., Geschäftsführer

Ärztlicher Leiter der Abteilung Rheumatologie im Krankenhaus Neuwittelsbach in München, Schwerpunktpraxis für Rheumatologie und Gastroenterologie
Schlüsselwörter

- Spondylitis ankylosans

- Patientenbefragung

- stationäre und medikamentöse Therapie

Key words

- ankylosing spondylitis

- patient survey

- in-patient and drug therapy

Bibliografie

DOI $10.1055 / \mathrm{s}-2008-1027173$

Akt Rheumatol 2008; 33:

53-59 @ Georg Thieme Verlag

KG Stuttgart · New York .

ISSN 0341-051X

Korrespondenzadresse

Prof. Dr. Ernst Feldtkeller

Michaeliburgstr. 15

81671 München

E.Feldtkeller@t-online.de

\section{Zusammenfassung \\ $\nabla$}

Hintergrund: In der Mitgliederzeitschrift der Deutschen Vereinigung Morbus Bechterew e.V. vom Dezember 2005 wurde ein 5-seitiger Fragebogen veröffentlicht mit 33 Fragen zur Diagnose, zur Krankheitsaktivität, zur stationären und medikamentösen Therapie sowie zu den Auswirkungen der neuesten Gesundheitsreformgesetze. Der Fragebogen wurde von 908 Patienten beantwortet. Ein Vergleich mit den Ergebnissen 11/2 Jahre früher ermöglicht Rückschlüsse auf gegenwärtige Trends in der Therapie der ankylosierenden Spondylitis (AS).

Ergebnisse: Bei einem Drittel der Patienten ist die AS mit einer anderen Grunderkrankung assoziiert: bei $19 \%$ mit einer Psoriasis, bei 13\% mit einer entzündlichen Darmerkrankung und bei $5 \%$ mit einer reaktiven Arthritis. Unter den Begleitmanifestationen war die periphere Oligoarthritis mit $71 \%$ besonders häufig, gefolgt von Enthesitiden bei $41 \%$ und Uveitis bei $30 \%$ der Patienten. Im Jahr 2005 wurden 8\% der Patienten in einem Akutkrankenhaus behandelt. Im Jahr 2004 waren es noch $10 \%$. In jedem der beiden Jahre suchten 15\% der Patienten eine Rehabilitationseinrichtung auf. Nur 44\% der Patienten hatten jemals an einer krankheitsspezifischen Patientenschulung teilgenommen, und zwar durchschnittlich bei 3,2 Rehabilitationsmaßnahmen mit durchschnittlich 7,9 Schulungsstunden. An einer Rehabilitationsmaßnahme, die auch Radon-Therapie beinhaltete, hatten $28 \%$ der Patienten (32\% der männlichen und $22 \%$ der weiblichen Patienten) mindestens einmal teilgenommen, und zwar durchschnittlich bei 5,9 Rehabilitationsaufenthalten. 74\% dieser Patienten hatten das Radon in einem Stollen inhaliert, 34\% hatten (ausschließlich oder auch) Radon-Wannenbäder genommen. Die Hälfte der Patienten nahm im Jahr 2005 regelmäßig antirheumatische Medikamente, ein weiteres

\section{Abstract \\ $\nabla$}

Background: In the membership journal of the Ankylosing Spondylitis Patient Organisation in Germany (Deutsche Vereinigung Morbus Bechterew, DVMB) of December 2005 a questionnaire was published with 33 questions concerning diagnosis, disease activity, therapy as in-patients and drug therapy. The questionnaire was returned by 908 patients. A comparison with responses to another questionnaire $1 \frac{1}{2}$ years earlier allows to draw conclusions about present trends in the therapy of ankylosing spondylitis (AS).

Results: In about one third of the patients AS is associated with another disease: In 19\% with psoriasis, in $13 \%$ with inflammatory bowel disease and in 5\% with reactive arthritis. Among the accompanying symptoms peripheral oligoarthritis was most frequent ( $71 \%$ of the patients), followed by enthesitis (41\%) and uveitis (30\%). In the year $20058 \%$ of the patients were treated in a hospital for acute episodes, compared to $10 \%$ in the year 2004. In each of the two years $15 \%$ of the patients were treated in a rehabilitation facility. Among all patients $44 \%$ had participated in a disease-specific patient education, and this in 3.2 rehabilitation courses with altogether 7.9 education hours on average. Radon therapy was received by $28 \%$ of the patients, on average in 5.9 rehabilitation courses. Among these patients $74 \%$ had inhaled the radon in a radon gallery, $34 \%$ had received (only or in addition) radon therapy in the form of radon baths. Antirheumatic drugs were taken regularly by $49 \%$ in the year 2005 , in addition by $32 \%$ occasionally, and $20 \%$ did not need any antirheumatics. In June 2004 only $12 \%$ of the respondents indicated to have taken no antirheumatics in the preceding 12 months. $60 \%\left(1 \frac{1}{2}\right.$ years earlier $\left.63 \%\right)$ were treated with non-steroidal anti-inflammatory drugs (NSAIDs), 15\% (27\%) with Coxibs, 14\% (17\%) with 
Drittel gelegentlich. Der Anteil, der 2005 ohne antirheumatische Medikamente auskam, betrug 20\%. Bei der Befragung im Juni 2004 gaben nur 12\% der Antwortenden an, in den 12 Monaten davor keine Antirheumatika benötigt zu haben. $60 \%$ der Antwortenden ( $11 / 2$ Jahre davor 63\%) wurden mit nichtsteroidalen Antirheumatika (NSAR) behandelt, 15\% (27\%) mit Coxiben, 14\% (17\%) mit Kortikosteroiden, 9\% (11\%) mit Sulfasalazin, 6,7 (5,7\%) mit Methotrexat, 8,5\% (5,5\%) mit TNF- $\alpha$-Inhibitoren, und $0,3 \%(0,8 \%)$ hatten sich einer Therapie mit Radium224-Chlorid unterzogen. Die Abbruchrate betrug bei den TNF$\alpha$-Inhibitoren $31 \%$, bei der Radium-224-Therapie nur $10 \%$. Eine mindestens 50\%ige Besserung der Schmerzen wurde bei 54\% der mit TNF- $\alpha$-Inhibitoren behandelten Patienten und bei $47 \%$ der mit Radium-224-Chlorid behandelten Patienten erreicht. Männliche und weibliche Patienten sowie Patienten mit unterschiedlichen Diagnosen unterschieden sich signifikant im Spektrum der in Anspruch genommenen Medikamente.

\section{Einleitung}

$\nabla$

Im Juni 2004 führten wir unter den Mitgliedern der Deutschen Vereinigung Morbus Bechterew e.V. (DVMB) eine Mitgliederbefragung durch, bei der neben der Diagnose und Klinik das Spektrum der verordneten Medikamente bei der ankylosierenden Spondylitis (AS) im Vordergrund des Interesses stand. Die Ergebnisse wurden im Jahre 2005 in dieser Zeitschrift veröffentlicht [19]. Seitdem hat sich in der Rheumatologie einiges verändert. Das COX-2-selektive Antirheumatikum Vioxx wurde wegen seiner unerwarteten Nebenwirkungen vom Markt genommen. Ein weiterer TNF-alpha-Blocker wurde für AS zugelassen. Die Produktion des radioaktiven Medikaments Radium224-Chlorid wurde wegen finanzieller Probleme des Herstellers stark gedrosselt und inzwischen so lange eingestellt, bis ein neuer Investor gefunden wird. In Deutschland kamen die beabsichtigten oder nicht beabsichtigten Folgen der Gesundheitspolitik noch hinzu. So lag es nahe, die Befragung $1 \frac{1}{2}$ Jahre später zu wiederholen, um zu eruieren, wie sich diese Veränderungen auf das Therapiespektrum einer definierten Patientengruppe auswirkten, und zwar wiederum nicht anhand des Patientenklientels ausgewählter Kliniken, sondern anhand des über das ganze Land verteilten Mitgliederstamms einer großen krankheitsspezifischen Patientenorganisation.

\section{Methode}

Die Fragebogen waren der DVMB-Mitgliederzeitschrift „Morbus-Bechterew-Journal“ beigeheftet $[27,28]$. Neben allgemeinen Fragen zum Alter und Geschlecht, zur Diagnose und zum Krankheitsverlauf enthielt der neue Fragebogen Fragen zu stationären Klinikaufenthalten und zur medikamentösen Therapie, auf die wir uns hier konzentrieren werden.

Die Antworten wurden von der Firma TNS-Emnid in zwei Datenbanken erfasst und von einem der Autoren (EF) mithilfe des Tabellenkalkulationsprogramms Excel ausgewertet. Die Signifikanz von Häufigkeitsunterschieden wurde nach der Methode des $\chi^{2}$-Tests für Vier-Felder-Tafeln (Fisher-Test) geprüft. Beim Vergleich von Mittelwerten wurde die Signifikanz durch den zweiseitigen t-Test mit unterschiedlichen Varianzen geprüft. corticosteroids, 9\% (11\%) with sulfasalacine, 6.7\% (5.7\%) with methotrexate, $8.5 \%$ (5.5\%) with TNF- $\alpha$ inhibitors, and $0.3 \%$ $(0.8 \%)$ had received injections of radium-224 chloride. The percentage of patients who interrupted the anti-TNF- $\alpha$ therapy was $31 \%$. For the radium-224 therapy the interruption rate was only $10 \%$. An improvement of pain by at least $50 \%$ was reported by $54 \%$ of patients treated with TNF- $\alpha$ inhibitors and by $47 \%$ of patients treated with radium-224 chloride. There were significant differences in the spectrum of medications taken between male and female patients and also between patients with different diagnoses.

\section{Antwortende Patienten \\ $\nabla$}

Innerhalb der vorgegebenen 6 Wochen nach Erscheinen des jeweiligen Zeitschriftenhefts wurden die Fragebogen von 1494 bzw. 908 Patienten beantwortet, also von 9,6 bzw. 5,9\% der Patientenmitglieder der DVMB. Bezüglich des Alters, der Krankheitsdauer, der Geschlechtsverteilung und der Krankheitsaktivität waren beide Kollektive vergleichbar ( $\bullet$ Tab.1). Auch im Anteil der Patienten mit assoziierten Erkrankungen und Begleitmanifestationen gab es keinen signifikanten Unterschied.

Tab. 1 Einige Charakteristika der antwortenden Patienten bei den DVMBPatientenbefragungen im Juni 2004 und im Dezember 2005 im Vergleich

\begin{tabular}{|l|ll|}
\hline Befragung im & Juni & Dezember \\
& $\mathbf{2 0 0 4}$ & $\mathbf{2 0 0 5}$ \\
\hline $\mathbf{n}=$ & $\mathbf{1 4 9 4}$ & $\mathbf{9 0 8}$ \\
\hline mittleres Alter bei der Befragung (Jahre) & 54,0 & 54,6 \\
\hline $\begin{array}{l}\text { mittleres Alter bei den ersten Symptomen } \\
\text { (Jahre) }\end{array}$ & 25,3 & 25,1 \\
\hline mittleres Alter bei der Diagnose (Jahre) & 35,3 & 34,8 \\
\hline mittlere Krankheitsdauer (Jahre) & 29,1 & 29,6 \\
\hline mittlere Diagnoseverzögerung (Jahre) & 10,4 & 9,7 \\
\hline Anteil weiblicher Patienten & $35 \%$ & $33 \%$ \\
\hline Anteil HLA-B27-positiver Patienten & $89 \%$ & $86 \%$ \\
\hline Anteil mit peripherer Gelenkbeteiligung & $64 \%$ & $71 \%$ \\
\hline Anteil mit Enthesitiden & nicht & $41 \%$ \\
\hline Anteil mit Uveitiden & gefragt & \\
\hline Anteil mit Osteoporose & $33 \%$ & $30 \%$ \\
\hline $\begin{array}{l}\text { primäre Spondylitis ankylosans (ohne asso- } \\
\text { ziierte Erkrankungen) }\end{array}$ & nicht & $20 \%$ \\
\hline $\begin{array}{l}\text { sekundäre Spondylitis ankylosans (mindes- } \\
\text { tens eine assoziierte Erkrankung) }\end{array}$ & $35 \%$ & $67 \%$ \\
\hline $\begin{array}{l}\text { darunter Psoriasis } \\
\text { darunter eine entzündliche Darmerkran- } \\
\text { kung (Morbus Crohn oder Colitis ulcerosa) }\end{array}$ & $19 \%$ & $13 \%$ \\
\hline darunter reaktive Arthritis & $5 \%$ & $5 \%$ \\
\hline
\end{tabular}




\section{Ergebnisse}

\section{Stationäre Klinikaufenthalte}

30\% der Antwortenden gaben im Dezember 2005 an, in den Jahren 2004 oder 2005 mindestens einmal wegen der AS stationär behandelt worden zu sein. In welchem Ausmaß darin Aufenthalte in einem Akutkrankenhaus enthalten sind und in welchem Umfang Rehabilitationsmaßnahmen in Anspruch genommen wurden, ist der $\bullet$ Tab. 2 zu entnehmen.

Vergleicht man die Angaben für die Kalenderjahre 2004 und 2005, so ergibt sich, dass die Einweisungen in ein Akutkrankenhaus tendenziell (allerdings nicht signifikant) zurückgingen, während die Häufigkeit von Rehabilitationsaufenthalten unverändert blieb. Betrachtet man die Entwicklung jedoch differenziert, so zeigt sich bei Patienten mit peripherer Gelenkbeteiligung ein signifikanter Rückgang der Akutkrankenhausaufenthalte. Auch die Akutkrankenhausaufenthalte der Patienten mit einer primären AS (ohne assoziierte Erkrankung) gingen signifikant zurück, während alle übrigen in $\bigcirc$ Tab. 2 erkennbaren Veränderungen nicht signifikant waren.

\section{Patientenschulung}

Die Deutsche Gesellschaft für Rheumatologie (DGRh) entwickelte für einige rheumatische Erkrankungen krankheitsspezifische Schulungsprogramme, darunter auch für die AS $[12,13]$. Das Schulungsprogramm für die AS wurde erfolgreich evaluiert. Es wirkt sich positiv auf das Wissen, die Einstellung und das Verhalten der Patienten aus und bringt auch gesundheitsökonomisch Vorteile [5]. Auch die Empfehlungen der Assessment in Ankylosing Spondylitis international working group (ASAS) enthalten den Hinweis auf die Patientenschulung als wichtigen Bestandteil der AS-Therapie [35]. Trotzdem wird die mit den Kostenträgern vereinbarte DGRh-Patientenschulung noch längst nicht von allen Rehabilitationseinrichtungen angeboten. Dies kommt auch im Ergebnis unserer Studie zum Ausdruck. Unter den antwortenden Patienten hatten nur 44\% mindestens einmal anlässlich einer Rehabilitationsmaßnahme an einer MorbusBechterew-spezifischen Patientenschulung teilgenommen, und zwar durchschnittlich bei 3,2 Reha-Maßnahmen. Die Dauer der Schulung betrug bei diesen Patienten insgesamt durchschnittlich 7,9 Stunden, also jedes Mal durchschnittlich 21/2 Stunden. Mehr als die Hälfte der antwortenden Patienten hatte also noch nie an einer krankheitsspezifischen Patientenschulung teilgenommen. Manche Rehabilitationseinrichtungen bieten nur eine allgemeine Informationsstunde über gesunde Lebensweise für alle Patienten der Klinik gemeinsam an, bei der auf die Probleme einzelner Krankheiten nicht eingegangen wird und bei der auch ein krankheitsspezifischer Erfahrungsaustausch nicht zustande kommen kann.

\section{Radon-Therapie}

Wie in $[11,32]$ gezeigt, gehört die Radon-Therapie zu den wenigen nachhaltig wirksamen Therapieformen bei der AS (in einem Radon-Stollen behandelte AS-Patienten hatten noch $1 / 2$ bis 1 Jahr nach der Behandlung im Mittel signifikant weniger Schmerzen als Patienten einer Vergleichsgruppe, die an einer vergleichbaren Rehabilitationsmaßnahme ohne Radon teilgenommen hatten, und benötigten entsprechend weniger Medikamente).

Unter den Antwortenden unserer Befragung im Dezember 2005 hatten $28 \%$ (32\% der männlichen und 22\% der weiblichen Patienten) mindestens einmal an einer Radon-Therapie teilgenommen ( $\bullet$ Tab.3). Die männlichen Patienten nahmen im Mittel seit 16,6 Jahren mehr oder weniger regelmäßig eine Radon-Therapie in Anspruch, und zwar durchschnittlich alle 2,6 Jahre ( $\bullet$ Tab.3). Bei weiblichen Patienten liegt die erste Radon-Kur im Mittel 11,5 Jahre zurück. Im zeitlichen Abstand

Tab.2 Stationäre Klinikaufenthalte wegen der AS unter 907 Patienten, die diese Fragen im Dezember 2005 beantworteten

\begin{tabular}{|c|c|c|c|c|c|c|c|}
\hline & $\begin{array}{l}\text { alle } \\
\text { Patienten }\end{array}$ & $\begin{array}{l}\text { rein } \\
\text { axiale } \\
\text { AS }\end{array}$ & $\begin{array}{l}\text { mit peripherer } \\
\text { Gelenkbeteiligung }\end{array}$ & $\begin{array}{l}\text { primäre } \\
\text { AS }\end{array}$ & $\begin{array}{l}\text { mit } \\
\text { Psoriasis- } \\
\text { Arthritis }\end{array}$ & $\begin{array}{l}\text { mit entzündlicher } \\
\text { Darmerkrankung }\end{array}$ & $\begin{array}{l}\text { mit re- } \\
\text { aktiver } \\
\text { Arthritis }\end{array}$ \\
\hline Anzahl Antwortende & 907 & 263 & 644 & 609 & 175 & 121 & 47 \\
\hline 2004 in einem Akutkrankenhaus & $10 \%$ & $5 \%$ & $12 \%$ & $10 \%$ & $9 \%$ & $8 \%$ & $19 \%$ \\
\hline 2005 in einem Akutkrankenhaus & $8 \%$ & $5 \%$ & $9 \%$ & $6 \%$ & $12 \%$ & $12 \%$ & $21 \%$ \\
\hline $\begin{array}{l}2004 \text { in einer Reha- } \\
\text { bilitationseinrichtung }\end{array}$ & $15 \%$ & $13 \%$ & $16 \%$ & $15 \%$ & $13 \%$ & $16 \%$ & $11 \%$ \\
\hline $\begin{array}{l}2005 \text { in einer Reha- } \\
\text { bilitationseinrichtung }\end{array}$ & $15 \%$ & $15 \%$ & $14 \%$ & $15 \%$ & $14 \%$ & $16 \%$ & $13 \%$ \\
\hline $\begin{array}{l}\text { kein stationärer } \\
\text { Aufenthalt wegen AS }\end{array}$ & $70 \%$ & $74 \%$ & $68 \%$ & $69 \%$ & $74 \%$ & $70 \%$ & $68 \%$ \\
\hline
\end{tabular}

Tab.3 Inanspruchnahme von Radon-Behandlungen jemals und im Jahr 2005

\begin{tabular}{|c|c|c|c|c|}
\hline & alle Patienten & männliche Antwortende & weibliche Antwortende & $\mathbf{p}$ \\
\hline jemals eine Radon-Therapie genommen & $28 \%$ & $32 \%$ & $22 \%$ & $<0,01$ \\
\hline durchschnittlich seit ... Jahren & 15 & 17 & 11 & $<0,001$ \\
\hline mittlere Zahl der Aufenthalte im Radon-Kurort & 5,9 & 6,3 & 4,8 & n.s. \\
\hline - daraus resultierender mittlerer Abstand (Jahre) & 2,6 & 2,6 & 2,4 & n.s. \\
\hline Radon-Therapie im Jahre 2005 & $10 \%$ & $11 \%$ & $6 \%$ & $<0,05$ \\
\hline - davon in einem Radon-Stollen & $74 \%$ & $74 \%$ & $72 \%$ & n.s. \\
\hline - und zwar an durchschnittlich ... Tagen & 11 & 10 & 12 & n.s. \\
\hline - davon Radon-Wannenbäder & $34 \%$ & $33 \%$ & $39 \%$ & n. s. \\
\hline - und zwar an durchschnittlich ... Tagen & 16 & 16 & 17 & n. s. \\
\hline
\end{tabular}


zwischen aufeinander folgenden Aufenthalten in einem Radon-Kurort unterschieden sich weibliche Patienten kaum von männlichen Patienten.

Im Jahr 2005 nahmen 10\% aller Antwortenden (11\% der männlichen und 6\% der weiblichen Patienten) eine Radon-Therapie in Anspruch ( Tab.3). Rund drei Viertel dieser Patienten inhalierte das Radon in einem Radon-Stollen, und zwar durchschnittlich an 11 Tagen. Rund ein Drittel nahm stattdessen oder außerdem Radon-Wannenbäder, und zwar an durchschnittlich 16 Tagen (• Tab.3).

\section{Verordnete Medikamente}

Bei unserer neuen Befragung gaben 49\% der Patienten an, in den vergangenen 12 Monaten regelmäßig antirheumatische Medikamente verwendet zu haben. Vor $1^{1} / 2$ Jahren [19] waren es noch $54 \%(p<0,05)$. Ein weiteres Drittel der Patienten nahm (wie vor $1 \frac{1}{2}$ Jahren) Antirheumatika gelegentlich. $20 \%$ der Patienten bekamen im Jahr 2005 keine Antirheumatika verschrieben, gegenüber $12 \%$ in 2003/04 ( $p<0,001$ ). Der Rückgang hing nicht vom Geschlecht oder von der Schwere der Erkrankung ab, wobei die periphere Gelenkbeteiligung als Maß für die Schwere der Erkrankung verwendet wurde. Die Krankheitsaktivität (Bath AS Disease Activity Index [4, 7, 23]) ist unter dem Einfluss der medikamentösen Therapie kein Maß für die Schwere der Erkrankung, während eine periphere Gelenkbeteiligung allgemein als ungünstiges Vorzeichen für einen schweren Krankheitsverlauf gilt [3, 9].

Der schwerere Krankheitsverlauf bei peripherer Gelenkbeteiligung spiegelt sich auch im umfangreicheren Medikamentenbedarf wider ( Tab.4). Im Vordergrund der medikamentösen ASTherapie stehen weiterhin die nichtsteroidalen Antirheumatika (NSAR). Ihr Gebrauch ging gegenüber 2003/04 nur geringfügig zurück. Während 2003/04 fast ein Drittel aller Morbus-Bechterew-Patienten (auch oder nur) COX-2-selektive Antirheumatika verwendeten [19], ging deren Anteil in der neuen Befragung um fast die Hälfte zurück. Ein leichter Rückgang war auch beim Gebrauch von Kortikosteroiden zu verzeichnen.

Der Gebrauch langsam wirkender Antirheumatika (Basismedikamente, meist abgekürzt als DMARDs von englisch Disease Modifying Anti-Rheumatic Drugs) blieb gegenüber 2003/04 nahezu unverändert. Innerhalb dieser Medikamentengruppe verschob sich aber die Verteilung leicht zugunsten des Methotrexat (nicht signifikant). Sulfasalazin blieb jedoch das am häufigsten verwendete DMARD, nicht nur bei einer peripheren Gelenkbeteiligung, sondern auch beim rein axialen Verlauf der AS.

Signifikant zugenommen hat der Anteil der TNF- $\alpha$-Inhibitoren, unter allen Antwortenden von 5,5\% auf 8,5\%. Wie die $\bullet$ Tab. 4 zeigt, ist dieser Anstieg wiederum unabhängig vom Geschlecht und von der Schwere der Erkrankung.

Unter den antwortenden Patienten gaben 10,2\% an, jemals mit einem TNF- $\alpha$-Hemmer behandelt worden zu sein. Wie sich diese Patienten auf die drei derzeit verfügbaren TNF- $\alpha$-Inhibitoren verteilen, ist in Tab. 5 eingetragen. Die Medikamente sind in der Reihenfolge aufgeführt, in der sie in Deutschland für die AS zugelassen wurden. Da manche Patienten nacheinander mit mehr als einem dieser Medikamente behandelt wurden, ist die Summe der Anteile der einzelnen Medikamente größer als der Anteil aller jemals mit einem TNF- $\alpha$-Hemmer behandelten Patienten.

\section{Wirksamkeit und Verträglichkeit der TNF- $\alpha$-Inhibitoren aus Patientensicht}

Ergebnisse zur Wirksamkeit und Verträglichkeit der TNF$\alpha$-Hemmer sind in der $\bullet$ Tab. 6 wiedergegeben. $31 \%$ der mit TNF- $\alpha$-Hemmern behandelten AS-Patienten brachen die Therapie ab, zum Teil wegen zu geringer Wirkung, zum Teil aber auch wegen der Nebenwirkungen. Die Abbruchrate war bei einer Verknüpfung der AS mit Psoriasis, mit einer entzündlichen

Tab.4 In den vergangenen 12 Monaten regelmäßig oder gelegentlich verwendete antirheumatische Medikamente unter allen 901 Patienten, die diese Frage im Dezember 2005 beantworteten, und zum Vergleich unter allen 1479 Patienten, die diese Fragen im Juni 2004 beantwortet hatten (Mehrfachnennungen zugelassen)

\begin{tabular}{|c|c|c|c|c|c|c|c|c|c|c|c|}
\hline & \multicolumn{3}{|c|}{ alle Antwortenden } & \multicolumn{2}{|c|}{ männlich } & \multicolumn{2}{|c|}{ weiblich } & \multicolumn{2}{|c|}{ rein axiale AS } & \multicolumn{2}{|c|}{ mit peripherer Arthritis } \\
\hline & 2004 & 2005 & $\mathbf{p}$ & 2004 & 2005 & 2004 & 2005 & 2004 & 2005 & 2004 & 2005 \\
\hline $\mathrm{n}=$ & 1479 & 901 & & 961 & 607 & 517 & 293 & 540 & 262 & 939 & 639 \\
\hline NSAR & $63 \%$ & $60 \%$ & n.s. & $63 \%$ & $60 \%$ & $62 \%$ & $61 \%$ & $58 \%$ & $52 \%$ & $66 \%$ & $64 \%$ \\
\hline Coxibe & $27 \%$ & $15 \%$ & $<0,001$ & $24 \%$ & $13 \%$ & $34 \%$ & $20 \%$ & $22 \%$ & $10 \%$ & $30 \%$ & $18 \%$ \\
\hline Kortikosteroide & $17 \%$ & $14 \%$ & n.s. & $14 \%$ & $12 \%$ & $24 \%$ & $19 \%$ & $11 \%$ & $10 \%$ & $21 \%$ & $16 \%$ \\
\hline Sulfasalazin & $11 \%$ & $9 \%$ & n.s. & $10 \%$ & $8 \%$ & $15 \%$ & $13 \%$ & $9 \%$ & $6 \%$ & $13 \%$ & $11 \%$ \\
\hline Methotrexat & $6 \%$ & $7 \%$ & n.s. & $5 \%$ & $5 \%$ & $8 \%$ & $11 \%$ & $4 \%$ & $4 \%$ & $7 \%$ & $8 \%$ \\
\hline Goldsalze & $0,3 \%$ & $0,3 \%$ & n.s. & $0,2 \%$ & $0,2 \%$ & $0,6 \%$ & $0,7 \%$ & $0,2 \%$ & $0,4 \%$ & $0,4 \%$ & $0,3 \%$ \\
\hline DMARDs insges. & $16 \%$ & $16 \%$ & n.s. & $14 \%$ & $13 \%$ & $21 \%$ & $24 \%$ & $13 \%$ & $10 \%$ & $18 \%$ & $19 \%$ \\
\hline Radium-224 & $0,8 \%$ & $0,3 \%$ & n.s. & $0,8 \%$ & $0,3 \%$ & $0,8 \%$ & $0,3 \%$ & $0,2 \%$ & $0,0 \%$ & $1,2 \%$ & $0,5 \%$ \\
\hline TNF-Inhibitoren & $5,5 \%$ & $8,5 \%$ & $<0,01$ & $4,6 \%$ & $7,4 \%$ & $7,2 \%$ & $11 \%$ & $5 \%$ & $8 \%$ & $6 \%$ & $9 \%$ \\
\hline keine Antirheum. & $12 \%$ & $20 \%$ & $<0,001$ & $14 \%$ & $22 \%$ & $9 \%$ & $15 \%$ & $19 \%$ & $32 \%$ & $8 \%$ & $15 \%$ \\
\hline
\end{tabular}

Tab. 5 Verteilung der jemals mit einem TNF- $\alpha$-Inhibitor behandelten Patienten auf die derzeit verfügbaren Medikamente dieser Medikamentengruppe (Mehrfachnennungen zugelassen)

\begin{tabular}{|c|c|c|c|}
\hline & alle Antwortenden & ohne Begleitmanifestationen & mit Begleitmanifestationen \\
\hline $\begin{array}{l}\text { jemals mit einem TNF- } \alpha \text {-Inhibitor behandelt } \\
\text { (Stand Dezember 2005), davon }\end{array}$ & $10,2 \%$ & $4,3 \%$ & $10,9 \%$ \\
\hline - mit Infliximab & $6,2 \%$ & $3,2 \%$ & $6,5 \%$ \\
\hline - mit Etanercept & $5,0 \%$ & $1,1 \%$ & $5,4 \%$ \\
\hline - mit Adalimumab & $0,8 \%$ & $0,0 \%$ & $0,9 \%$ \\
\hline
\end{tabular}


Darmerkrankung oder mit reaktiver Arthritis signifikant größer als bei Patienten mit einer primären AS. Besonders groß war die Abbruchrate mit $71 \%$ bei Patienten, bei denen die AS mit einer reaktiven Arthritis assoziiert war. Bei Patienten mit einer primären AS lag die Abbruchrate bei 20\% ( $\bullet$ Tab.6). Bei 54\% aller mit einem TNF- $\alpha$-Inhibitor behandelten Patienten hat sich die Stärke der Schmerzen um mehr als 50\% gebessert. Bei Patienten mit einer primären Spondylitis ankylosans waren es sogar $62 \%$, bei Patienten mit einer reaktiven Arthritis dagegen nur $29 \%$.

Wirksamkeit und Verträglichkeit der Therapie mit Radium-224-Chlorid aus Patientensicht

Dieselben Fragen zur Wirksamkeit und Verträglichkeit wie bei der Anti-TNF- $\alpha$-Therapie wurden auch zur Therapie mit Radium-
224-Chlorid gestellt. Die Radium-224-Therapie ist neben der Neurokognitiven Therapie [18, 20, 21] die einzige Therapieform, von der in der Literatur berichtet wird, dass dadurch AS-Patienten eine über viele Jahre anhaltende Besserung ohne dauernde Weiterbehandlung erfuhren [1, 26, 29-31]. Wie Jahn [26] in einer umfangreichen Studie nachwies, wird auch das Fortschreiten der Wirbelsäulenkyphose durch die Radium-224-Therapie verlangsamt. Die kurzzeitige Wirksamkeit wurde kürzlich anhand der heute international üblichen Parameter untersucht [2].

Die Ergebnisse unserer Befragung zur Radium-Therapie sind in der $\odot$ Tab. 7 wiedergegeben. Insgesamt ließen sich unter den 908 antwortenden Patienten 49 (5,4\%) mit Radium-224 behandeln, davon 22 Patienten in den Jahren 1947 bis 1987 und 28 Patienten in den Jahren 1995 bis 2005 (ein Patient ließ sich 1986 und nochmals 2005 damit behandeln).

Tab. 6 Anteil der Patienten, welche die Anti-TNF- $\alpha$-Therapie abbrachen, und Gründe für das Absetzen des Medikaments. Mittlere Stärke der Schmerzen vor der Therapie, zwei Monate später und zum Zeitpunkt der Befragung sowie Anteil der Patienten, bei denen die Schmerzen um mindestens $50 \%$ reduziert wurden

\begin{tabular}{|c|c|c|c|c|c|}
\hline & $\begin{array}{l}\text { alle Pa- } \\
\text { tienten }\end{array}$ & $\begin{array}{l}\text { primäre Spondylitis } \\
\text { ankylosans }\end{array}$ & $\begin{array}{l}\text { mit Psoriasis- } \\
\text { Arthritis }\end{array}$ & $\begin{array}{l}\text { mit entzündlicher } \\
\text { Darmerkrankung }\end{array}$ & $\begin{array}{l}\text { mit reaktiver } \\
\text { Arthritis }\end{array}$ \\
\hline $\begin{array}{l}\text { Anzahl jemals damit behandelter } \\
\text { Patienten }\end{array}$ & 93 & 60 & 15 & 15 & 7 \\
\hline $\begin{array}{l}\text { Anteil an allen Patienten mit dieser } \\
\text { Diagnose }\end{array}$ & $10 \%$ & $10 \%$ & $9 \%$ & $12 \%$ & $15 \%$ \\
\hline Therapie abgebrochen & $31 \%$ & $20 \%$ & $47 \%$ & $47 \%$ & $71 \%$ \\
\hline - wegen Wirkungslosigkeit & $12 \%$ & $10 \%$ & $13 \%$ & $7 \%$ & $29 \%$ \\
\hline - wegen Nebenwirkungen & $15 \%$ & $10 \%$ & $20 \%$ & $20 \%$ & $43 \%$ \\
\hline - aus anderen Gründen & $9 \%$ & $5 \%$ & $13 \%$ & $20 \%$ & $14 \%$ \\
\hline weiterhin damit behandelt & $69 \%$ & $80 \%$ & $53 \%$ & $53 \%$ & $29 \%$ \\
\hline $\begin{array}{l}\text { durchschnittliche Schmerzen vor } \\
\text { Behandlungsbeginn } \\
\text { (0= keine Schmerzen, } \\
10=\text { unerträgliche Schmerzen) }\end{array}$ & 8,2 & 8,2 & 8,1 & 8,4 & 8,0 \\
\hline $\begin{array}{l}\text { durchschnittliche Schmerzen } 2 \text { Monate } \\
\text { nach Behandlungsbeginn }\end{array}$ & 3,5 & 3,5 & 3,9 & 3,6 & 4,0 \\
\hline $\begin{array}{l}\text { durchschnittliche Schmerzen zum } \\
\text { Zeitpunkt der Befragung }\end{array}$ & 3,4 & 3,5 & 3,0 & 2,9 & 3,0 \\
\hline $\begin{array}{l}\text { Anteil mit mindestens } 50 \% \text { iger } \\
\text { Besserung unter allen Behandelten }\end{array}$ & $54 \%$ & $62 \%$ & $40 \%$ & $47 \%$ & $29 \%$ \\
\hline
\end{tabular}

\begin{tabular}{|c|c|c|c|}
\hline & $\begin{array}{l}\text { alle } \\
\text { Patienten }\end{array}$ & $\begin{array}{l}\text { mit peripherer } \\
\text { Gelenkbeteiligung }\end{array}$ & rein axiale $A S$ \\
\hline Anzahl jemals damit behandelter Patienten & 49 & 37 & 12 \\
\hline Anteil an allen Patienten dieser Gruppe & $5,4 \%$ & $5,7 \%$ & $4,6 \%$ \\
\hline davon die Therapie vorzeitig abgebrochen & $10 \%$ & $14 \%$ & $0 \%$ \\
\hline - wegen Wirkungslosigkeit & $2 \%$ & $3 \%$ & $0 \%$ \\
\hline - wegen Nebenwirkungen & $2 \%$ & $3 \%$ & $0 \%$ \\
\hline - aus anderen Gründen & $6 \%$ & $8 \%$ & $0 \%$ \\
\hline alle 10 - 12 Injektionen bekommen & $90 \%$ & $86 \%$ & $100 \%$ \\
\hline $\begin{array}{l}\text { durchschnittliche Schmerzen vor Behandlungsbeginn } \\
\text { ( } 0 \text { = keine Schmerzen, } 10 \text { = unerträgliche Schmerzen) }\end{array}$ & 7,8 & 7,8 & 7,7 \\
\hline Schmerzen nach der letzten Injektion & 4,2 & 4,2 & 4,2 \\
\hline Schmerzen in der Woche vor der Befragung & 4,0 & 4,1 & 3,7 \\
\hline $\begin{array}{l}\text { Anteil mit mindestens } 50 \% \text { iger Besserung } \\
\text { unmittelbar nach der letzten Injektion }\end{array}$ & $43 \%$ & $47 \%$ & $33 \%$ \\
\hline $\begin{array}{l}\text { Anteil mit mindestens } 50 \% \text { iger Besserung } \\
\text { bis zur Befragung unter allen Behandelten }\end{array}$ & $47 \%$ & $49 \%$ & $42 \%$ \\
\hline $\begin{array}{l}\text { mittlere Dauer seit Durchführung der Therapie bei } \\
\text { Patienten mit mindestens } 50 \% \text { iger Besserung }\end{array}$ & 17 Jahre & 18 Jahre & 14 Jahre \\
\hline
\end{tabular}

Tab. 7 Anteil der Patienten, die die Therapie mit Radium224-Chlorid abbrachen, und Gründe für das Absetzen des Medikaments. Mittlere Stärke der Schmerzen vor und nach der Therapie, Anteil der Patienten, bei denen die Schmerzen um mindestens $50 \%$ reduziert wurden, und mittlere Dauer seit Durchführung der Therapie bei diesen Patienten 
Fünf dieser Patienten (10\%) brachen die Behandlungsserie, die normalerweise aus 10-12 Injektionen in wöchentlichem Abstand besteht, vorzeitig ab, darunter einer wegen Wirkungslosigkeit, einer wegen Nebenwirkungen und drei aus „sonstigen“ Gründen.

Auch bei der Therapie mit Radium-224 hat sich die Stärke der Schmerzen bei fast der Hälfte aller behandelten Patienten um mehr als 50\% gebessert. Der Unterschied in der Ansprechquote zwischen Anti-TNF- $\alpha$-Therapie und Radium-224-Therapie ist statistisch nicht signifikant.

\section{Diskussion}

$\nabla$

Eine Schwäche der Studie ist zweifellos die geringe Rücklaufquote von 9,6 bzw. 5,9\%, die damit zusammenhängen dürfte, dass beide Fragebogen nicht persönlich zugeschickt wurden, sondern in der DVMB-Mitgliederzeitschrift veröffentlicht wurden. Offensichtlich haben viele Mitglieder nicht realisiert, dass in der Heftmitte ein Fragebogen abgedruckt war, oder sich nicht persönlich aufgefordert gefühlt, die Fragen zu beantworten. Bei der Befragung von 1996 [14-17], als eine Zufallsauswahl von 3000 Patienten persönlich angeschrieben wurde und nach 3 Wochen an zufällig ausgewählte Säumige ein Erinnerungsschreiben verschickt wurde, betrug die Rücklaufquote 54\%. Angesichts der großen Zahl ausgefüllter Fragebogen können wir jedoch davon ausgehen, dass auch diesmal ein repräsentativer Querschnitt der AS-Patienten erfasst wurde und nicht die Anhänger einer bestimmten Therapieform (z.B. eines bestimmten Medikaments) unter den Antwortenden überrepräsentiert sind.

Ein Motiv für den Abdruck des Fragebogens war die Frage, wie weit sich der Zugang zu stationären Heilbehandlungen und der Gebrauch antirheumatischer Medikamente in diesen $1 \frac{1}{2}$ Jahren veränderte, da einerseits die Politik zunehmend Einfluss auf die Verschreibungspraxis zu nehmen versucht, andererseits die medikamentöse Rheuma-Therapie sich derzeit durch die Einführung der TNF- $\alpha$-hemmenden Medikamente im Umbruch befindet.

Der Zugang zu stationären Heilbehandlungen war schon vor 2003 erschwert worden. Ihre Inanspruchnahme blieb im Beobachtungszeitraum stabil.

Leider kam mehr als die Hälfte der Patienten noch nie in den Genuss einer krankheitsspezifischen Patientenschulung. Dies dürfte mit dem steigenden Kostendruck auf die Rehabilitationseinrichtungen zusammenhängen. Umso wichtiger ist das Informationsangebot der DVMB für diejenigen Patienten, die ihr als Mitglied beigetreten sind. Und auch Patienten, die sich zu diesem Schritt nicht entschließen konnten (schätzungsweise 90\% aller diagnostizierter AS-Patienten in Deutschland), haben wenigstens die Möglichkeit, über das Internet an einen Teil dieses für Patienten aufbereiteten Informationsangebots heranzukommen und auf diese Weise von den DVMB-Aktivitäten zu profitieren.

Der Erfahrungsaustausch unter den DVMB-Mitgliedern dürfte auch dazu geführt haben, dass ein beträchtlicher Teil von ihnen von der Möglichkeit der Radon-Therapie Gebrauch macht oder wenigstens den Versuch gemacht hat, davon zu profitieren, obwohl Strahlenanwendungen in den Medien immer wieder für Negativmeldungen genutzt werden. Insofern ist es möglich, dass die Nutzer der Radon-Therapie in dem von uns befragten Kollektiv überrepräsentiert sind.
Dass bei weiblichen Patienten die erste Radon-Kur im Mittel weniger weit zurückliegt als bei männlichen Patienten ( $\bullet$ Tab.3), überrascht zunächst, ist aber kein Wunder, denn die weiblichen Antwortenden sind im Mittel 5 Jahre jünger, wahrscheinlich wegen der häufigeren Fehldiagnosen und der im Mittel längeren Diagnoseverzögerung bei Frauen in früheren Jahrzehnten [16, 17], sodass auch die Diagnose bei gleichem gegenwärtigem Alter im Mittel weniger weit zurückliegt.

Dass der Gebrauch COX-2-selektiver Antirheumatika in den $1^{1 / 1} 2$ Jahren um fast die Hälfte zurückging, hängt sicher mit der Rücknahme von Vioxx nach den Meldungen über aufgetretene HerzKreislauf-Nebenwirkungen zusammen. Der Rückgang bei der Nutzung antirheumatischer Medikamente insgesamt dürfte mit den Restriktionen im Gesundheitswesen zusammenhängen. Auch eine gesteigerte Aufmerksamkeit auf Medikamentennebenwirkungen könnte hier eine Rolle spielen. Beunruhigend ist, dass sich unter den Patienten mit peripherer Gelenkbeteiligung, also einem relativ schweren Krankheitsverlauf, der Anteil, der keine Medikamente verschrieben bekam (oder sich keine Medikamente verschreiben ließ), nahezu verdoppelt hat.

Der Geschlechtsunterschied in der Inanspruchnahme von Coxiben ( Tab.4) findet sich auch in der Kerndokumentation der Regionalen Kooperativen Rheumazentren [25] wieder, dort allerdings weniger stark ausgeprägt als in unseren Daten. Auch der Geschlechtsunterschied beim Einsatz von Kortikosteroiden ist in unserem Kollektiv stärker ausgeprägt als in der Kerndokumentation. Die häufigere Behandlung weiblicher AS-Patienten mit TNF- $\alpha$-Inhibitoren widerlegt besonders eindrucksvoll das Gerücht vom „milderen“ und häufig „abortiven“ Verlauf bei weiblichen AS-Patienten, denn die Notwendigkeit dieser Medikamente wird wegen der hohen Kosten besonders sorgfältig überprüft. Darauf, dass dieser „mildere“ Verlauf bei weiblichen AS-Patienten nur die Progression der Ankylose betrifft, während weibliche Patienten vor allem nach langer Krankheitsdauer stärker mit Schmerzen zu kämpfen haben als ihre männlichen Mitpatienten, wurde bereits in früheren Arbeiten hingewiesen $[15,25,34]$.

Im Zusammenhang mit der hohen Abbruchrate der Anti-TNF$\alpha$-Therapie bei der mit einer reaktiven Arthritis assoziierten AS, verbunden mit einer vergleichsweise hohen Nebenwirkungsrate und einer vergleichsweise geringen Erfolgsrate bei dieser Patientengruppe, ist daran zu erinnern, dass die TNF$\alpha$-Inhibitoren zur Behandlung einer reaktiven Arthritis noch nicht zugelassen sind. Dass sich TNF- $\alpha$-Inhibitoren bei unterschiedlichen Spondyloarthritiden unterschiedlich gut bewährt haben (und es darin erhebliche Unterschiede zwischen den verfügbaren Präparaten gibt), ist in der Literatur ausgiebig berichtet worden $[6,8]$. Angaben über Therapieerfolge mit TNF$\alpha$-Inhibitoren bei der reaktiven Arthritis reichen aber über Spekulationen kaum hinaus [10]. In einer Studie, deren Ergebnisse 2005 veröffentlicht wurden [22], wurde berichtet, dass es bei 7 Patienten mit reaktiver Arthritis unter Etanercept zu einer Besserung der Beschwerden und der Gewebebefunde kam, jedoch nicht zu einem Verschwinden der Beschwerden und einer Normalisierung der Gewebebefunde. Entgegen früherer Befürchtungen kam es dabei zu keiner Reaktivierung der Infektion. Insofern scheint die Befürchtung mindestens in Bezug auf Etanercept dieser kleinen Studie zufolge unbegründet zu sein.

In Anbetracht der Tatsache, dass es zur Therapie mit Radium-224 noch immer keine Studie gibt, die heutigen Maßstäben genügt, ist das Patientenurteil zu dieser Therapieform von be- 
sonderem Interesse. Dass die Radium-224-Therapie bei einem ähnlich hohen Anteil der Patienten wie die Anti-TNF- $\alpha$-Therapie zu einer mindestens 50\%igen Besserung führt ( $\bullet$ Tab.6, $7)$, bestätigt die in der Literatur [1, 2, 26, 29-31] mitgeteilten positiven Erfahrungen bezüglich Wirksamkeit dieses Medikament. Die signifikant geringere Abbruchrate im Vergleich zur Anti-TNF- $\alpha$-Therapie bestätigt auch die in der Literatur [2, 24, 34] berichtete gute Verträglichkeit der Radium-224-Therapie. Vor allem die Rate der Therapieabbrüche wegen Nebenwirkungen beträgt nur einen Bruchteil von derjenigen bei der AntiTNF- $\alpha$-Therapie. Ein weiterer wesentlicher Unterschied könnte in der Nachhaltigkeit der Radium-224-Therapie bestehen, die sich aber durch unsere Befragung nur unsicher quantifizieren lässt.

Der Rückgang der Radium-224-Therapie in den $1{ }^{1} / 2$ Jahren zwischen den beiden Befragungen dürfte damit zusammenhängen, dass die Herstellerfirma dieses Medikaments die Produktion aus finanziellen Gründen einschränken musste.

Insgesamt bietet unser Ansatz eine interessante Möglichkeit, Trends in der Therapie der AS aufzuspüren und sich dabei nicht auf die Perspektive der behandelnden Ärzte zu verlassen, sondern die Sicht der Betroffenen selbst zu erfassen.

\section{Interessenkonflikt: Nein}

\section{Literatur}

1 Adam WE. Geschichtliche Entwicklung der Therapie mit Radium-224 bei der Spondylitis ankylosans. Aktuelle Rheumatologie 1987; 12 (Sonderheft 1): 13-14

2 Alberding A, Stierle H, Brandt J et al. Wirksamkeit und Verträglichkeit von Radiumchlorid in der Behandlung der ankylosierenden Spondylitis. Z Rheumatol 2006; 65: 245-251

3 Amor B, Santos RS, Nahal $R$ et al. Predictive factors for the longterm out-come of spondyloarthropathies. J Rheumatol 1994; 21: 1883-1887

4 Bönisch A, Ehlebracht-König I. Der BASDAI-D - ein Fragebogen zur Erfassung der Krankheitsaktivität bei Spondylitis ankylosans und verwandten Erkrankungen. Z Rheumatol 2003; 62: 251-263

5 Bönisch A, Ehlebracht-König I. Die Akzeptanz des Schulungsprogramms der DGRh für Patienten mit Spondylitis ankylosans und verwandten Spondylarthropathien. Akt Rheumatol 2003; 28: 254-263

6 Brandt J, Sieper J, Braun J. Die Therapie der ankylosierenden Spondylitis und der undifferenzierten Spondyloarthritis mit TNF-alpha-Antagonisten. Z Rheumatol 2003; 62: 218-227

7 Brandt J, Westhoff G, Rudwaleit $M$ et al. Validierung einer deutschen Version des Fragebogens BASDAI zur Messung der Krankheitsaktivität bei ankylosierender Spondylitis. Z Rheumatol 2003; 62: 264-273

8 Braun J, de Keyser F, Brandt J et al. New treatment options in spondyloarthropathies: increasing evidence for significant efficacy of anti-tumor necrosis factor therapy. Curr Opin Rheumatol 2001; 13: 245-249

9 Braun J, Pincus T. Mortality, course of disease and prognosis of patients with ankylosing spondylitis. Clin Exp Rheumatol 2002; 20 (Suppl 28): S16-S22

10 Butrimiene I, Jarmalaite S, Ranceva J et al. Different cytokine profiles in patients with chronic and acute reactive arthritis. Rheumatology 2004; 43: 1300-1304

11 Deetjen $P$, Falkenbach A, Jöckel H. Indikationen und kontrollierte klinische Studien. In: Radon-Dokumentations- und Informationszentrum Schlema e.V (Hrsg). Radon als Heilmittel. Hamburg: Verlag Dr. Kovač, 2005

12 Ehlebracht-König I, Bönisch A. Das Schulungsprogramm der DGRh für Patienten mit Spondylitis ankylosans und verwandten Spondylarthropathien - Programmaufbau. Akt Rheumatol 2003; 28: 243-253
13 Ehlebracht-König I, Bönisch A. Patientenschulungen in der Rehabilitation von Patienten mit chronischen Polyarthritiden und Spondylarthritiden. Akt Rheumatol 2004; 29: 248-254

14 Feldtkeller E. Unterschiede im Krankheitsverlauf männlicher und weiblicher Spondylarthritis-Patienten. Akt Rheumatol 1998; 23: 145-153

15 Feldtkeller E. Rückgang von Spondylarthritis-Schmerzen nach langer Krankheitsdauer begünstigt männliche Patienten. Akt Rheumatol 1998; 23: 176-181

16 Feldtkeller E. Erkrankungsalter und Diagnoseverzögerung bei Spondylarthropathien. Z Rheumatol 1999; 58: 21-30

17 Feldtkeller E, Lemmel EM. Zur Situation von Spondyloarthritis-Patienten. Ergebnisse einer Repräsentativbefragung der DVMB. Nürnberg: Novartis Pharma Verlag, 1999

18 Feldtkeller E. Spondylitis-ankylosans-Therapie im Wandel. Naturheilpraxis 2002; 12: 1773-1777

19 Feldtkeller E, Hammel L, Kellner H. Krankheitsaktivität und medikamentöse Therapie bei Spondylitis ankylosans - Ergebnisse einer Patientenbefragung. Akt Rheumatol 2005; 30: 223-231

20 Feldtkeller E, Kellner $H$. Neurocognitive therapy of ankylosing spondylitis: final results of pilot study indicate neuronal component of pathogenesis. Clin Exp Rheumatol 2006; 24: 478

21 Feldtkeller E, Kellner H. Neurokognitive Therapie der Spondylitis ankylosans: Pilotstudie zur Ermittlung der Erfolgsrate. Arthitis + Rheuma 2007; 27: 279-284

22 Flagg SD, Meador R, Hsia E et al. Decreased pain and synovial inflammation after etanercept therapy in patients with reactive and undifferentiated arthritis: an open-label trial. Arthritis Rheum 2005; 53: 613-617

23 Garrett S, Jenkinson T, Kennedy G et al. A new approach to defining disease status in ankylosing spondylitis: the Bath Ankylosing Spondylitis Disease Activity Index. J Rheumatol 1994; 21: 2286-2291

24 Glöbel B. Nutzen-Risiko-Betrachtung verschiedener Therapieformen der Spondylitis ankylosans. Aktuelle Rheumatologie 1987; 12 (Sonderheft 1): 38-41

25 Huscher D, Thiele K, Zink A. Patienten mit rheumatoider Arthritis und ankylosierender Spondylitis: Geschlechtsspezifische Unterschiede in der Behandlung. arthritis+rheuma 2004; 24: 205-210

26 Jahn K. Spondylitis ankylosans: Spätergebnisse nach Radium-224und Kinesiotherapie. Aktuelle Rheumatologie 1987; 12 (Sonderheft 1): $30-32$

27 Kellner H, Hammel L. Fragebogen zur Versorgungssituation der Morbus-Bechterew-Patienten in Deutschland. Morbus-Bechterew-Journal Nr. 97, Gelbe Seiten in der Heftmitte Juni 2004

28 Kellner H, Feldtkeller E, Hammel L. Fragebogen 2006 zur Versorgungssituation der Morbus-Bechterew-Patienten in Deutschland. MorbusBechterew-Journal Nr. 103, Seiten mit farbigem Rand in der Heftmitte Dezember 2005

29 Knop J, Stritzke P, Heller M et al. Ergebnisse einer Radium224-Therapie der ankylosierenden Spondylitis (M. Strümpell-Marie-Bechterew). Z Rheumatol 1982; 41: 272-275

30 Koch W. Indikationsstellung und Ergebnisse einer Radium-224-(Thorium X-)Therapie der Spondylitis ankylopoetica (Sp.a.). Z Orthop 1978; 116: 608-616

31 Lemmel EM. Diagnose und Therapie der ankylosierenden Spondylitis. Osteoporose \& Rheuma aktuell 2003; 1: 28-31

32 van Tubergen A, Landewé $R$, van der Heijde D et al. Combined spa-exercise therapy is effective in patients with ankylosing spondylitis: a randomized controlled trial. Arthritis Care Res 2001; 45: 430-438

33 Wick RR, Nekolla EA. Long term investigation of late effects in ankylosing spondylitis patients treated with Radium-224. GSF-Bericht 06/05. Neuherberg: GSF - Forschungszentrum für Umwelt und Gesundheit, 2005

34 Zink A, Braun J, Listing J et al. German Collaborative Arthritis Centers: Disability and handicap in rheumatoid arthritis and ankylosing spondylitis - results from the German rheumatological database. J Rheumatol 2000; 27: 613-622

35 Zochling J, van der Heijde D, Burgos-Vargas R et al. ASAS/EULAR recommendations for the management of ankylosing spondylitis. Ann Rheum Dis 2006; 65: 442-452 